\title{
Antagonizing $\mathrm{P}_{2} \mathrm{Y}_{12}$ Receptor Inhibitors: Current and Future Options
}

\author{
Dietmar Trenk ${ }^{1} \quad$ Laura Hille $^{1} \quad$ Stefan Leggewie $^{1} \quad$ Christian Stratz $^{1} \quad$ Thomas G. Nührenberg $^{1}$ \\ Daniel Aradi ${ }^{2,3}$ Karsten Schrör ${ }^{4}$ Dirk Sibbing ${ }^{5,6}$
}

\footnotetext{
${ }^{1}$ Department of Cardiology and Angiology II, Clinical Pharmacology, University Heart Centre Freiburg, Bad Krozingen, Germany

${ }^{2}$ Heart Center Balatonfured, Balatonfured, Hungary

${ }^{3}$ Heart and Vascular Center, Semmelweis University, Budapest, Hungary

${ }^{4}$ Institute for Pharmacology and Clinical Pharmacology, University Hospital Düsseldorf, Düsseldorf, Germany

5 Department of Cardiology, LMU München, Munich, Germany

${ }^{6}$ DZHK (German Centre for Cardiovascular Research), partner site

Munich Heart Alliance, Munich, Germany
}

Address for correspondence Dietmar Trenk, PhD, Department of Cardiology and Angiology II - Clinical Pharmacology, University Heart Centre Freiburg - Bad Krozingen, Südring 15, D-79189 Bad Krozingen, Germany (e-mail: Dietmar.Trenk@universitaets-herzzentrum.de).

Thromb Haemost 2019;119:1606-1616.
Platelet activation and aggregation play an important role in the development of ischemic events during and after acute coronary syndromes (ACSs) and percutaneous coronary interventions (PCIs). ${ }^{1}$ Acetylsalicylic acid (aspirin) was the first antiplatelet drug with proven benefit in ACS. ${ }^{2-4}$ Studies demonstrating significant platelet activation in ACS and during PCI despite treatment with aspirin and intense anti- coagulant regimens stimulated clinical studies investigating novel antithrombotic regimens using two antiplatelet drugs, namely aspirin and a thienopyridine. Compared with aspirin plus anticoagulation (heparin followed by vitamin $\mathrm{K}$ antagonists), dual antiplatelet therapy (DAPT) with aspirin and ticlopidine which was the only available thienopyridine at that time decreased the incidence of cardiac and vascular complications after the placement of coronary artery stents. received

May 8, 2019

accepted after revision

June 13, 2019 (c) 2019 Georg Thieme Verlag KG Stuttgart · New York
DOI https://doi.org/

$10.1055 / \mathrm{s}-0039-1693738$. ISSN 0340-6245. 
Moreover, the incidence of bleeding complications during follow-up was markedly decreased. ${ }^{5}$ DAPT with aspirin and a $\mathrm{P} \mathrm{Y}_{12}$ receptor inhibitor became therefore the standard of care for prevention of ischemic complications in ACS patients and in patients undergoing $\mathrm{PCI}$.

Ticlopidine was replaced early by the second generation thienopyridine clopidogrel due to safety concerns regarding allergy, skin, or gastrointestinal disorders. ${ }^{6,7}$ Clopidogrel has a similar degree of $\mathrm{P}_{2} \mathrm{Y}_{12}$ inhibition and bleeding risk like ticlopidine but an improved safety profile. Clinical studies have demonstrated that DAPT with aspirin and clopidogrel results in a substantial rate of patients with attenuated response to clopidogrel impacting on clinical outcome. ${ }^{8-12}$ Large-scale clinical outcome studies combining aspirin with the $\mathrm{P}_{12} \mathrm{Y}_{12}$ receptor antagonists prasugrel or ticagrelor in patients presenting with an ACS demonstrated a prognostic benefit by a more consistent and more potent inhibition of the P2Y 12 receptor compared with DAPT with aspirin plus clopidogrel. ${ }^{13,14}$ The reduction in ischemic events was, however, achieved at the cost of an increased rate of major bleeding events due to the exaggerated suppression of platelet reactivity.

Current European and U.S. guidelines endorse the use of DAPT after PCI with stent placement or previous myocardial infarction. ${ }^{15,16}$ DAPT with aspirin and clopidogrel is prescribed for patients with stable coronary artery disease (SCAD); while DAPT combining aspirin with prasugrel or ticagrelor is favored over clopidogrel for patient presenting with an ACS.

The duration of DAPT in CAD depends on the clinical presentation of the patient (stable CAD vs. ACS), a clinical assessment of the postprocedural ischemic risk, and the potential bleeding risk. Use of risk scores to guide DAPT duration has been endorsed in the recently published focused update on DAPT in CAD of the European Society of Cardiology (ESC). ${ }^{17}$

Any decision of premature interruption of DAPT must be based on sound clinical judgment and a clear understanding of the potential risk and benefits, including acute thrombotic events. Therefore, current guidelines recommend to postpone elective surgical interventions beyond the early phase of coronary intervention and stenting, that is usually a minimum of 30 days after the procedure. ${ }^{16}$ This timeframe is based upon analysis of Danish population base registries assessing the risk associated with surgery among drugeluting stent-PCI-treated patients compared with patients with ischemic heart disease undergoing a similar surgical procedure. ${ }^{18}$ When stratified for time from PCI to surgery, surgery within the first month after PCI was associated with increased risk of ischemic events. Thus, elective surgery should be postponed to $>30$ days after $\mathrm{PCI}$ and controlled cessation of antiplatelet therapy before surgery according to the estimated time for recovery of platelet function, which is 3 days for the reversible inhibitor ticagrelor and 5 and 7 days for the irreversible inhibitors clopidogrel and prasugrel, should be performed.

However, there are clinical scenarios where strategies for rapid establishment of hemostasis by reversal of the anti- platelet effect are required. This comprises various emergency situations such as:

- Active bleeding, particularly severe or life-threatening bleeding into critical organs like intracranial bleeding or intraocular bleeding.

- Unscheduled, urgent, or emergent procedures that carry a presumed high bleeding risk, or procedures where the consequences of even minor bleeding would be unacceptable (e.g., spinal surgery or other neurosurgical procedures).

- Major trauma with bleeding.

No guidance based upon randomized clinical trials is available for patients on DAPT who either develop active bleeding complications or who are scheduled for urgent procedures with presumed high bleeding risk. Current ESC guidelines recommend to balance in this setting ischemic risk (e.g., indication for DAPT, time from last PCI with stenting) versus recurrent/prolonged bleeding risks. ${ }^{17}$ In severe bleeding, de-escalation of antiplatelet therapy from DAPT to single antiplatelet therapy should be considered, and if bleeding persists, stopping all antithrombotic medications is recommended. In life-threatening bleeding, all antithrombotic medications are discontinued immediately and red blood cell or platelet transfusion are mentioned as general recommendation. ${ }^{17}$

After the decision for reversal of platelet inhibition has been made, the question is how to achieve it effectively? The current evidence is discussed in this review which focuses on reversal strategies for antagonizing the antiplatelet effect of currently available oral $\mathrm{P}_{2} \mathrm{Y}_{12}$ inhibitors clopidogrel, prasugrel, and ticagrelor only.

There is no medical need for reversal strategies for the parenteral $\mathrm{P}_{2} \mathrm{Y}_{12}$ receptor cangrelor due to the rapid decline of platelet inhibition after stopping the infusion due to the short half-life of this compound ${ }^{19}$ (see - Table $\mathbf{1}$ ).

\section{Pharmacological Differences between Oral P2Y $_{12}$ Receptor Inhibitors}

- Table 1 summarizes the key pharmacological and pharmacokinetic data of the currently approved $\mathrm{P} \mathrm{Y}_{12}$ receptor inhibitors. There are substantial differences between the thienopyridine-type $\mathrm{P} \mathrm{Y}_{12}$ platelet inhibitors (clopidogrel, prasugrel) and the nonthienopyridine derivatives ticagrelor and cangrelor.

The parent compounds clopidogrel and prasugrel are prodrugs without any antiplatelet pharmacological effect and both compounds require metabolization to the active antiplatelet compound. Clopidogrel is activated via a twostep metabolism process in the liver with the intermediate metabolite being generated by CYP1A2, CYP2B6, and CYP2C19 and further metabolism catalyzed by four enzymes (CYP2B6, CYP2C9, CYP2C19, and CYP3A4).

The more consistent and faster metabolic formation of the active metabolite (AM) of prasugrel is due to the fact that the first metabolite is formed by esterases (human carboxylesterase 2) in the gut and plasma, followed by a single cytochrome (CYP)-dependent step with primary involvement of CYP3A 
Table 1 Clinical pharmacology of $\mathrm{P}_{2} \mathrm{Y}_{12}$ receptor inhibitors

\begin{tabular}{|c|c|c|c|c|}
\hline Property & Clopidogrel & Prasugrel & Ticagrelor & Cangrelor \\
\hline Receptor blockade & Irreversible & Irreversible & Reversible & Reversible \\
\hline Prodrug & Yes & Yes & No & No \\
\hline Administration route & Oral - Once daily & Oral - Once daily & Oral - Twice daily & IV - Bolus and infusion \\
\hline $\begin{array}{l}\text { Half-life of } \\
\text { parent drug }\end{array}$ & $\sim 6$ hours & $<5$ minutes & $6-12$ hours & 3-9 minutes \\
\hline $\begin{array}{l}\text { Half-life of active } \\
\text { metabolite }\end{array}$ & 30 minutes & $\begin{array}{l}\text { Distribution half-life: } \\
30-60 \text { minutes } \\
\text { Elimination half-life: } \\
2-15 \text { hours }\end{array}$ & $8-12$ hours & $\mathrm{N} / \mathrm{A}$ \\
\hline Binding site & ADP binding site & ADP binding site & Allosteric binding site & Allosteric binding site \\
\hline $\begin{array}{l}\text { Mode of antagonism } \\
\text { plasma protein }\end{array}$ & Noncompetitive & Noncompetitive & Noncompetitive & Semicompetitive \\
\hline $\begin{array}{l}\text { Plasma protein } \\
\text { binding }\end{array}$ & $\begin{array}{l}\text { C } 98 \% \\
\text { C-AM 94\% }\end{array}$ & $P>98 \%$ & $\begin{array}{l}\text { T } 99.8 \% \\
\text { T-AM 99.8\% }\end{array}$ & n.r. \\
\hline Onset of action & $2-8$ hours & $\begin{array}{l}30 \text { minutes to } \\
4 \text { hours }\end{array}$ & 30 minute to 4 hours & $\sim 2$ minutes \\
\hline Offset of action & $5-10$ days & $7-10$ days & $3-5$ days & 60 minutes \\
\hline CYP drug interaction & CYP2C19 & No & CYP3A4 to T-AM & No \\
\hline Approved settings & $\begin{array}{l}\text { ACS (invasively / } \\
\text { noninvasively managed), } \\
\text { stable CAD PCI, PAD, } \\
\text { and ischemic stroke }\end{array}$ & $\begin{array}{l}\mathrm{PCl} \text { in patients } \\
\text { with ACS }\end{array}$ & $\begin{array}{l}\text { ACS (invasively or } \\
\text { noninvasively managed) } \\
\text { or history of MI }\end{array}$ & $\begin{array}{l}\mathrm{PCl} \text { in patients with } \\
\text { or without } \mathrm{ACS}\end{array}$ \\
\hline
\end{tabular}

Abbreviations: ACS, acute coronary syndrome; ADP, adenosine diphosphate; AM, active metabolite; CAD, coronary artery disease; IV, intravenous; $\mathrm{PAD}$, peripheral arterial disease; $\mathrm{PCl}$, percutaneous coronary intervention; TAM, ticagrelor's active metabolite. Source: Table modified from Sillén et al and Ferri et al. ${ }^{20,21}$

and CYP2B6, and only partial contribution of CYP2C9 and CYP2C19. Furthermore, the pharmacological profiles of the AMs of both drugs are characterized by two distinct features: (1) they inhibit the platelet $\mathrm{P}_{2} \mathrm{Y}_{12}$ receptor irreversibly, that is, platelet inhibition persists for the life-span of the platelet and (2) their half-lives are in the order of 30 minutes (clopidogrel) and 7 hours (prasugrel). ${ }^{20}$

In contrast, the cyclopentyl-triazolo-pyrimidine derivative ticagrelor is an adenosine triphosphate (ATP) analogue with the parent compound binding reversibly in a noncompetitive manner to the $\mathrm{P}_{2} \mathrm{Y}_{12}$ receptor at distinct sites to adenosine diphosphate (ADP) and the thienopyridines. Ticagrelor undergoes CYP3A4-mediated metabolism to an AM (AR-C124910XX), and the metabolite inhibits also the P2Y 12 receptor in a reversible manner. Thus, in the case of ticagrelor, half-life of elimination of parent drug and the AM (which both are in the range of 6-12 hours) determine the recovery of platelet function after cessation of drug administration. Furthermore, both ticagrelor and its AM ARC124910XX are highly bound to plasma proteins (> 99.8\%), that is, unbound fraction is less than $0.2 \% .^{21}$

\section{Effect of Platelet Supplementation on Restoration of Platelet Function}

Based upon the lack of a specific antidote for reversal of platelet inhibition by the currently used $\mathrm{P}_{2} \mathrm{Y}_{12}$ inhibitors, a variety of in vitro and in vivo experiments were performed aiming to assess the effect of noninhibited platelet supplementation on platelet reactivity.

\section{Clopidogrel}

Vilahur et al administered loading doses of aspirin (325 mg) and clopidogrel (300-600 mg) to healthy subjects followed by short-term maintenance dosing with aspirin (81 mg/day) and clopidogrel $(75 \mathrm{mg} / \text { day })^{22}$ Platelet-rich plasma (PRP) was prepared from blood samples drawn at 4 and 72 hours after starting treatment with the latter time point corresponding to 24 hours after ingestion of the last maintenance dose of both antiplatelets. Platelet inhibition by aspirin/clopidogrel was determined by agonist-induced light transmission aggregometry (LTA) and flow cytometric analysis of glycoprotein IIb/ IIla receptor expression using various stimulants. To normalize platelet reactivity in PRP from the treated subjects, increasing amounts of pooled platelets (PPs) from five untreated volunteers were added ex vivo to the PRP obtained from the treated subjects. Addition of 40 to $50 \%$ of PRP from untreated subjects normalized platelet reactivity assessed after stimulation with $10 \mu \mathrm{M}$ ADP in LTA and further addition of $10 \%$ untreated platelets fully normalized aggregation. The authors estimated that transfusion of 10 platelet concentrate units (which was deemed equivalent to in vitro addition of $40 \%$ untreated PRP) after a 300-mg clopidogrel loading or 12.5 units (50\% untreated PRP) after a 600-mg loading may adequately normalize clopidogrel-induced platelet inhibition. 
The transfer of the amount of platelet concentrates required for restoration of platelet function in patients on DAPT with aspirin and clopidogrel estimated in ex vivo experiments into clinical practice is challenged by results from a study using a design which is closer to the clinical setting. ${ }^{23}$ Prüller et al investigated in healthy subjects if transfusion of stored autologous platelets restores low platelet reactivity on DAPT (aspirin/clopidogrel). Two autologous platelet concentrates were obtained from each study participant. Thereafter, an antiplatelet regimen was started. Loading doses of aspirin $300 \mathrm{mg}$ and clopidogrel $300 \mathrm{mg}$ were administered followed by maintenance daily dosing of aspirin $100 \mathrm{mg}$ and clopidogrel $75 \mathrm{mg}$ on days 2 and 3. Two stored autologous platelet concentrates were transfused 24 hours after last dosing of the antiplatelets on day 4. A nearly complete recovery of platelet reactivity index (PRI) determined with vasodilator-stimulated phosphoprotein phosphorylation (VASP kit, Biocytex, Marseille, France) was observed after transfusion of the second platelet concentrate with a slight attenuation of effect 24 hours thereafter. However, assessment of platelet reactivity by LTA after stimulation with arachidonic acid and ADP shows hardly no immediate response to platelet transfusion and a partial recovery of platelet reactivity not earlier than 24 hour post-transfusion.

\section{Prasugrel}

Research on platelet function normalization was extended to DAPT with the combination of aspirin and prasugrel. ${ }^{24,25}$

Subjects pretreated with a single dose of aspirin $325 \mathrm{mg}$ received subsequently a loading dose of prasugrel $60 \mathrm{mg}^{24}$ The experimental setting tried to estimate the earliest time after a prasugrel loading dose when added platelets are no longer inhibited by prasugrel's AM considering the longer halflife of elimination of the AM compared with clopidogrel's AM. Serial blood samples were obtained at 2, 6, 12, and 24 hours postprasugrel for platelet reactivity after stimulation with 20 $\mu \mathrm{M}$ ADP by LTA and VerifyNow PRUTest (Werfen, Barcelona, Spain) and simultaneous determination of the plasma concentration of the AM of prasugrel. At each time point, fresh concentrated platelets from untreated donors were added ex vivo to the blood samples from treated subjects. The protocol aimed to raise the platelet counts by 0 (control), 40, 60, and $80 \%$. Concentration-dependent increases in platelet reactivity versus respective controls by both LTA and VerifyNow PRUTest were observed after in vitro supplementation with untreated donor platelets at each time point investigated ( $\mathbf{- F i g}$. 1). The augmented effect at 2 hours after the prasugrel loading dose was attributed to the still high plasma concentration of the AM of prasugrel at that time $(42.4 \pm 11 \mathrm{ng} / \mathrm{mL})$. A sharp increase in platelet reactivity by platelet supplementation was observed from 2 to 6 hours after loading and the effect was more or less stable thereafter. It seems that the plasma concentrations of the AM of prasugrel at 6 hours $(4.5 \pm 1 \mathrm{ng} / \mathrm{mL})$ are below the threshold for a detectable inhibition of the supplemented donor platelets. Thus, administration of platelet concentrates is most effective beyond 6 hours after the last administration of prasugrel, although partial normalization of prasugrel effects could be obtained earlier. ${ }^{24}$

\section{Ticagrelor}

Hobl et al administered a loading dose of ticagrelor $180 \mathrm{mg}$ to healthy subjects. ${ }^{26}$ Blood samples withdrawn 3 hours after dosing were spiked with autologous PRP prepared from blood collected before ticagrelor. Mixing increasing amounts of PRP with the post-dosing blood samples in ratios between $1: 10$ and 1:3 improved ex vivo determined ADP-induced platelet aggregation in a dose-dependent manner with marked interindividual variability. However, the predefined cut-off for restoration of platelet aggregation was achieved even at the highest platelet supplementation in 8 out of 20 subjects only.

Investigations in blood samples drawn 2 hours after dosing from patients on chronic DAPT with aspirin and ticagrelor confirmed a dose-dependent recovery of ADP-induced aggregation by increasing ex vivo platelet supplementation but it was less effective than in patients on aspirin plus clopidogrel. ${ }^{27}$

The most comprehensive study performed repeated blood sampling $(4,6,24$, and 48 hour after last dosing) in patients with cardiovascular disease after single bolus dosing of ticagrelor $180 \mathrm{mg}$ and aspirin $325 \mathrm{mg}$ as well as after maintenance treatment with ticagrelor $90 \mathrm{mg}$ twice daily and aspirin $81 \mathrm{mg}$ once daily. ${ }^{28}$ Patients' blood samples were supplemented with concentrated platelets from healthy donors in vitro aiming to raise platelet counts by 0 (control), 25,50 , and $75 \%$. The time course in decline of platelet inhibition was similar after loading as well as after cessation of maintenance therapy. Platelet supplementation restored platelet reactivity in a dose-dependent manner with the restoration of function being strongly dependent on the time elapsed since last dose administration. A small but statistically significant effect could be observed as early as 6 hours after last dosing, but the clinical relevance of this improvement seems questionable. Beyond 24 hours after last dosing, platelet transfusions (2-3 units) can be expected to substantially reverse the antiplatelet effect of ticagrelor. ${ }^{28,29}$

The time window between the last dose of the antiplatelet and blood sampling for assessment of the effect of platelet supplementation for reversal of platelet inhibition is of special interest for studies with ticagrelor due to the reversibility of the binding to the $\mathrm{P}_{2} \mathrm{Y}_{12}$ receptor, the long half-lives of parent compound and the AM in plasma, and the twice daily dosing of ticagrelor in maintenance treatment. This has been shown in a study, which exposes blood from untreated healthy volunteers with blood and plasma from patients on treatment with clopidogrel, prasugrel, or ticagrelor, respectively. ${ }^{30}$ Blood was drawn from the patients 3 hours after the dosing of the antiplatelets. When platelets from untreated subjects were stimulated after mixing with PRP from patients treated with different $\mathrm{P}_{2} \mathrm{Y}_{12}$ inhibitors, ADP-induced expression of P-selectin was not affected by PRP from clopidogrel- or prasugreltreated patients, while addition of PRP from ticagrelor-treated patients decreased surface expression of P-selectin. If blood from healthy subjects was spiked with plasma from patients on $\mathrm{P}_{2} \mathrm{Y}_{12}$ inhibitors, ADP-induced aggregation in multiple electrode aggregometry (MEA; Multiplate, Roche Diagnostics, Rotkreuz, Switzerland) was not affected by plasma from patients on clopidogrel, was slightly inhibited by plasma 


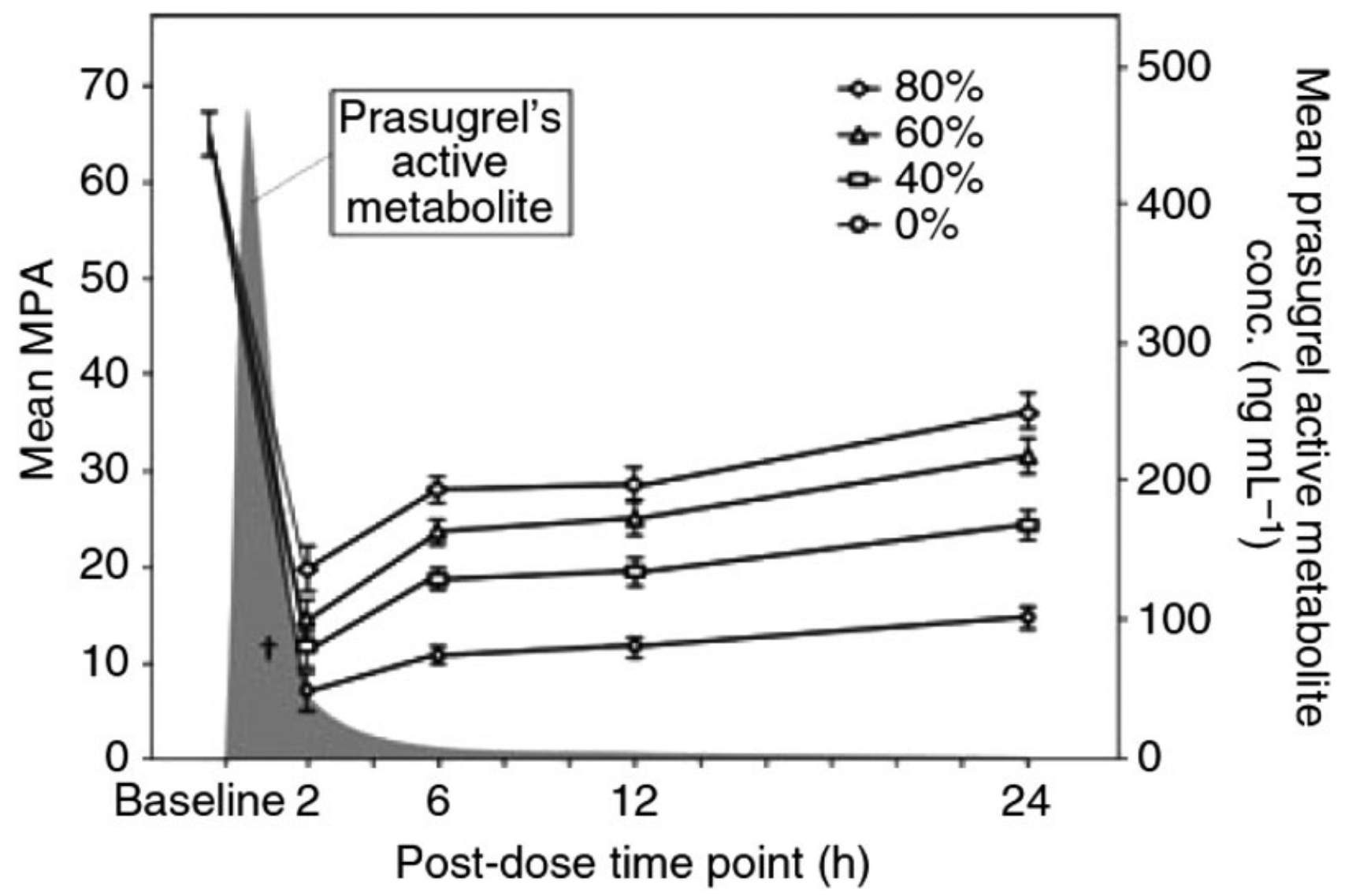

Fig. 1 Maximum platelet aggregation (MPA) to $20 \mu \mathrm{M}$ adenosine diphosphate (ADP) using light transmission aggregometry (LTA) (left $Y$-axis) and prasugrel active metabolite concentrations (right $Y$-axis, gray area) over 24 hours following a loading dose of prasugrel $60 \mathrm{mg}$. Platelet aggregation was measured in aspirin- and prasugrel-treated subjects' blood supplemented with (40,60, and $80 \%)$ and without (0\%) fresh platelets. Aggregation values in all supplemented samples are higher than respective controls $(0 \%, p \leq 0.01)$ except " $\dagger$ " but lower than baseline $(p<0.01)$. Aggregation values in supplemented samples at 6 hours are higher than respective values at 2 hours $(p<0.05$ for 40 and $60 \%)$, but similar to those at 12 hours ( $p=$ NS for all). Prasugrel active metabolite was not measured before the 2-hour time point; results are taken from an earlier study. ${ }^{42}$ Figure reproduced with permission from Zafar et al. ${ }^{24}$

from patients on prasugrel, and aggregation was markedly attenuated if plasma from ticagrelor patients was added. The results are in line with the expected plasma concentrations of the three antiplatelets and their AMs extrapolated from dosing regimens used and the time of blood sampling after dosing.

Schoener et al extended the reversal strategies for $\mathrm{P}_{2} \mathrm{Y}_{12^{-}}$ related platelet inhibition based upon platelet supplementation by an interesting approach considering the high plasma protein binding of ticagrelor and its AM. ${ }^{31}$ Patients on DAPT (aspirin plus guideline-recommended dosing with clopidogrel, prasugrel, or ticagrelor) with an ACS were enrolled. Blood samples were drawn at trough of the respective chronic dosing regimen and not earlier than 24 hours after loading. Platelet reactivity was assessed by VASP test. Supplementation with increasing amounts of freshly prepared PRP from healthy donors increased VASP PRI irrespective of the antiplatelet drug administered ( - Fig. 2). PPs obtained from the local blood bank restored platelet inhibition in clopidogrel and prasugrel samples but had surprisingly no effect on samples from ticagrelor-treated patients. Further experiments with samples from ticagrelor-treated patients investigated the reversal effect of sedimented PRP platelets which were resuspended in PPs buffer. No reversal effect could be determined although the same amounts of PRP or PP were administered (- Fig. 3). PRP preparations contain physiological concentrations of plasma proteins, while the PP concentrates provided from the local blood bank are free of relevant amounts of protein since they are stored in a special stabilizing buffer solution. This fact indicates that proteins-namely serum album-might contribute to the different effects on ticagrelor reversal observed between supplementation of PRP and PP. To test this hypothesis, increasing amounts of human serum albumin in the range of 8 to $80 \mathrm{~g} / \mathrm{L}$ were added to samples from ticagrelor-treated patients. Supplementation with serum albumin restored platelet inhibition by ticagrelor since VASP-PRI increased in a concentration-dependent manner (-Fig. 3). Similar investigations with the same concentration range of human serum albumin were performed with samples from clopidogrel- as well as prasugrel-treated patients. Platelet inhibition by clopidogrel and prasugrel was reversed less pronounced by supplementation of human serum albumin which might be attributed to the irreversible mode of receptor binding of clopidogrel and prasugrel. Thus, the approach of albumin supplementation for reversal of ticagrelor-induced platelet inhibition might be a possible and clinically applicable approach. The in vitro added concentrations of human serum 


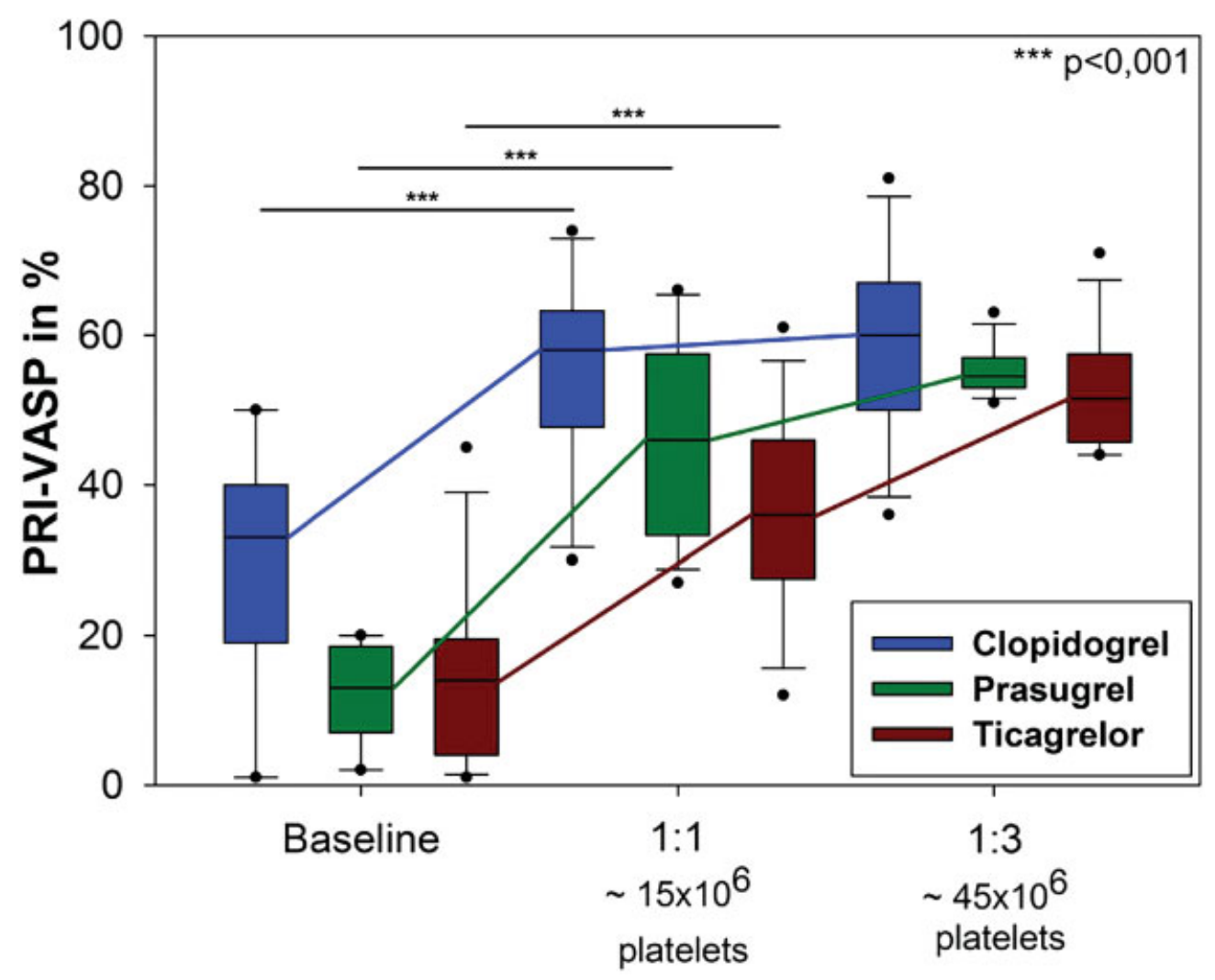

Fig. 2 Reversal of platelet inhibition by clopidogrel, prasugrel, and ticagrelor determined by vasodilator-stimulated phosphoprotein-platelet reactivity index (VASP-PRI) after ex vivo platelet supplementation using drug-free platelet-rich plasma (PRP). Figure reproduced with permission from Schoener et al. ${ }^{31}$

album correspond to doses of approximately 20 to $40 \mathrm{~g}$ human albumin in vivo. However, clinical data in patients with acute severe bleeding or patients scheduled for urgent surgical procedures are lacking so far.

The Action Study Group performed the APTITUDE study (Efficacy of Ex Vivo Autologous and In Vivo Platelet Transfusion in the Reversal of P2Y 12 Inhibition by Clopidogrel, Prasugrel, and Ticagrelor). ${ }^{32}$ APTITUDE-ACS enrolled patients presenting with an ACS or for elective PCI. Normalization of P2 $\mathrm{Y}_{12}$ inhibition by clopidogrel, prasugrel, and ticagrelor was investigated by ex vivo supplementation of autologous platelets. In their ex vivo experiments, PRP from treatment-naive subjects was mixed in increasing proportions $(30,50$, and $80 \%$ ) with PRP sampled 4 hours after loading doses of either clopidogrel $600 \mathrm{mg}$, clopidogrel $900 \mathrm{mg}$, prasugrel $60 \mathrm{mg}$, or ticagrelor $180 \mathrm{mg}$. In the in vitro experiments, the percentage restoration of residual platelet aggregation (assessed by LTA after stimulation with $20 \mu \mathrm{M}$ ADP) increased with increasing amounts of supplemented treatment-naive platelets. The finally achieved platelet reactivity obtained with $80 \%$ proportion supplemented platelets decreased with increasing potency of the $\mathrm{P}_{2} \mathrm{Y}_{12}$ receptor blocking intensity in the order clopidogrel $600 \mathrm{mg}<$ clopidogrel $900 \mathrm{mg}<$ prasugrel $60 \mathrm{mg}<$ ticagrelor $180 \mathrm{mg}$.

Patients with excessive bleeding undergoing cardiac surgery while on a maintenance dose of aspirin plus either clopidogrel, prasugrel, or ticagrelor were enrolled into the APTITUDE-Coronary Artery Bypass Graft (APTITUDE-CABG) substudy. ${ }^{32}$ Platelet reactivity was assessed by VASP reactivity index before and within 30 minutes after completion of the transfusion of platelet concentrates. The dose of the platelet concentrates was set according to a weight-based guideline (mean 5.5 concentrate units of platelets). While a statistically significant increase in VASP reactivity index (relative increase 23.1\%) was observed in patients on clopidogrel, only a small nonsignificant increase was observed in the patients on prasugrel/ticagrelor. Although the number of patients undergoing surgery on prasugrel/ticagrelor was small, one might speculate that the attenuated efficacy of the platelet transfusions might be attributed at least in part to the use of pooled buffy coat platelets in the majority of patients. ${ }^{31}$

A case report highlights the challenges in management of patients with life-threatening bleeding on DAPT with ticagrelor. ${ }^{33}$ A 65-year-old man on DAPT with aspirin and ticagrelor post-PCI with stent implantation was admitted with hemiplegia and hemispatial neglect. Twelve hours after thrombolysis with recombinant tissue plasminogen activator consciousness of the patient decreased and an intracranial hematoma with intraventricular hemorrhage and acute hydrocephalus requiring an external ventricular drain was diagnosed. The patient died shortly after surgery despite transfusion of 17 platelet concentrates aiming to reverse the effects of antiplatelet therapy. Platelet function assays performed before and after transfusion of platelet concentrates indicate significant attenuation of arachidonic acid-induced aggregation (VerifyNow Aspirin test), while no reversal of $\mathrm{P}_{2} \mathrm{Y}_{12}$ inhibition could be demonstrated neither by VerifyNow PRUTest nor by VASP-PRI. 


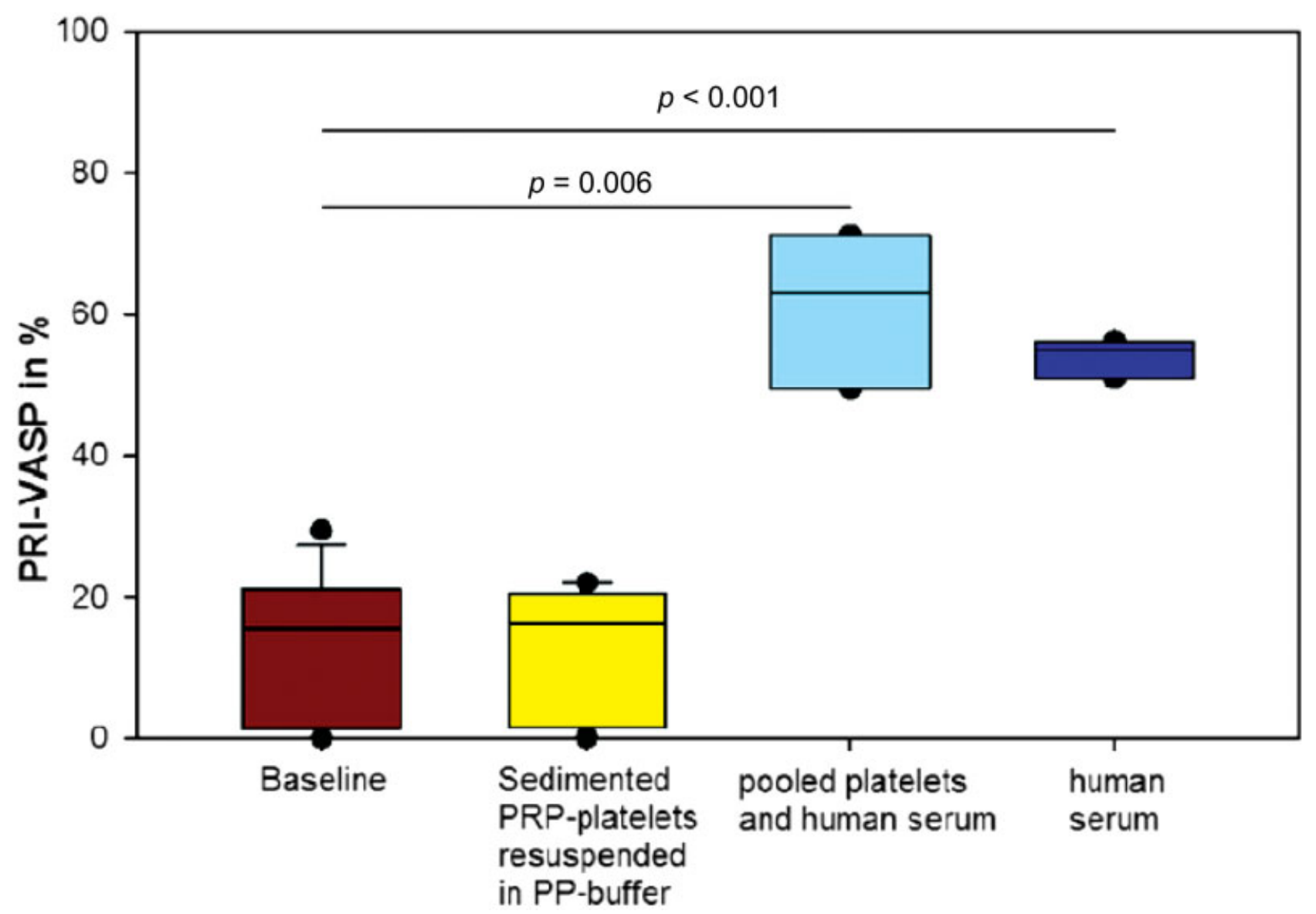

Fig. 3 Change in vasodilator-stimulated phosphoprotein-platelet reactivity index (VASP-PRI) after supplementation of platelet-rich plasma (PRP) platelets resuspended in pooled platelets (PPs) buffer, and PP with human serum and human serum alone in ticagrelor-treated patients. A significant VASP increase after adding of human serum with or without PP is evident. Figure reproduced with permission from Schoener et al. ${ }^{31}$

\section{Hemadsorption for Reversal of Ticagrelor}

Only in vitro data on the use of sorbent hemadsorption as an alternate method for removal of ticagrelor from blood are available so far. ${ }^{34}$ The styrene copolymer Porapak Q 50-80 mesh and CytoSorb were used to investigate the removal of ticagrelor from bovine serum albumin solutions, whereas CytoSorb was also used to remove the drug from human blood samples (-Fig. 4). The hemadsorption technique was highly effective in removing the drug ticagrelor, but no functional data on reversal of platelet inhibition are available so far. Besides this, several questions remain to be investigated at present. These comprise the efficiency of removal of the AM of ticagrelor as well as the suitability of the method for emergent situations when a slow removal is clinically unacceptable. Furthermore, the effect of hemadsorption on untended removal of other drugs or endogenous compounds needs to be investigated.

\section{Human Monoclonal Antigen-Binding Fragment PB2452 for Reversal of Ticagrelor}

PB2452 (former name MEDI2452) is an antigen-binding fragment (Fab) antidote for ticagrelor. ${ }^{35}$ The Fab has a 20 pM affinity for ticagrelor, which is 100 times stronger than ticagrelor's affinity for the platelet $\mathrm{P}_{2} \mathrm{Y}_{12}$ receptor. The binding characteristics for ticagrelor's AM (TAM) are similar. PB2452 is highly specific for ticagrelor/TAM and preclinical experiments excluded binding to adenosine, ATP, ADP, or a variety of structurally related drugs. The antidote neutralizes in a concentration-dependent manner free (unbound) ticagrelor/TAM in plasma (-Fig. 5), causes a dissociation of both compounds from the $\mathrm{P}_{2} \mathrm{Y}_{12}$ receptor to the plasma compartment enabling binding by the circulating Fab. This results in a PB2452 concentration-dependent reversal of ticagrelor/TAM-induced inhibition of human platelets (-Fig. 5). The Fab reverses ticagrelor-induced bleeding in a mice tail bleeding model. It was assumed from the preclinical data, that the amount of ticagrelor/TAM in a patient is neutralized completely within minutes after administration of PB2452. ${ }^{36}$

The further preclinical assessment included investigation of the hemostatic effect of PB2452 in a pig bleeding model after treatment of the animals with aspirin and ticagrelor. ${ }^{37}$ Administration of PB2452 completely neutralized free ticagrelor/ TAM within 5 minutes after administration of the Fab which translates into a gradual normalization of ADP-induced platelet aggregation and a numerical reduction in blood loss.

Most recently, the results of a first randomized, doubleblind, placebo-controlled phase 1 trial for assessment of dosefinding, safety, efficacy, and pharmacokinetics of PB2425 in healthy subjects pretreated with ticagrelor were published. ${ }^{38}$ 

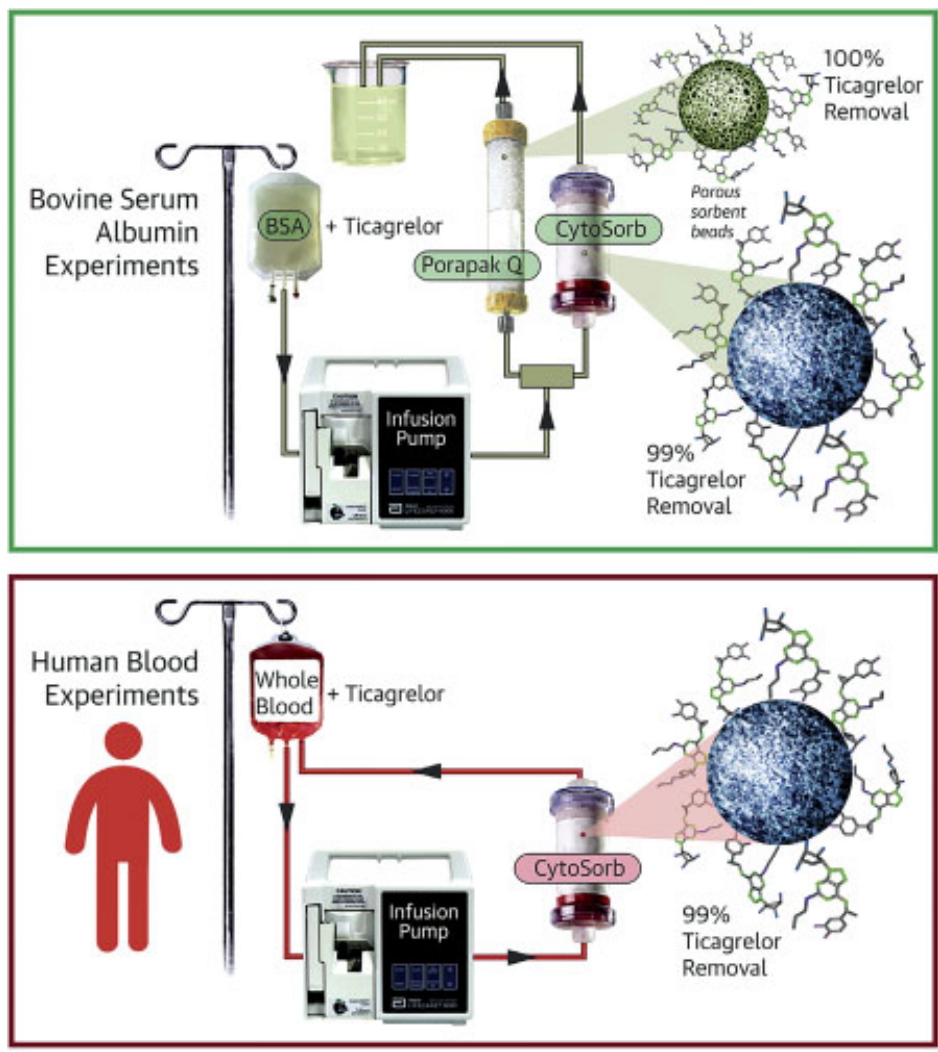

Fig. 4 Illustration of the experimental ex vivo setting of sorbent hemadsorption for ticagrelor removal. The upper panel illustrates the first-pass experiment using bovine serum albumin (BSA) solution preincubated with ticagrelor. The lower panel displays the recirculating technique used for human blood spiked with ticagrelor. Figure reproduced with permission from Angheloiu et al. ${ }^{34}$

\section{Ticagrelor and TAM induced inhibition of human platelets in vitro}

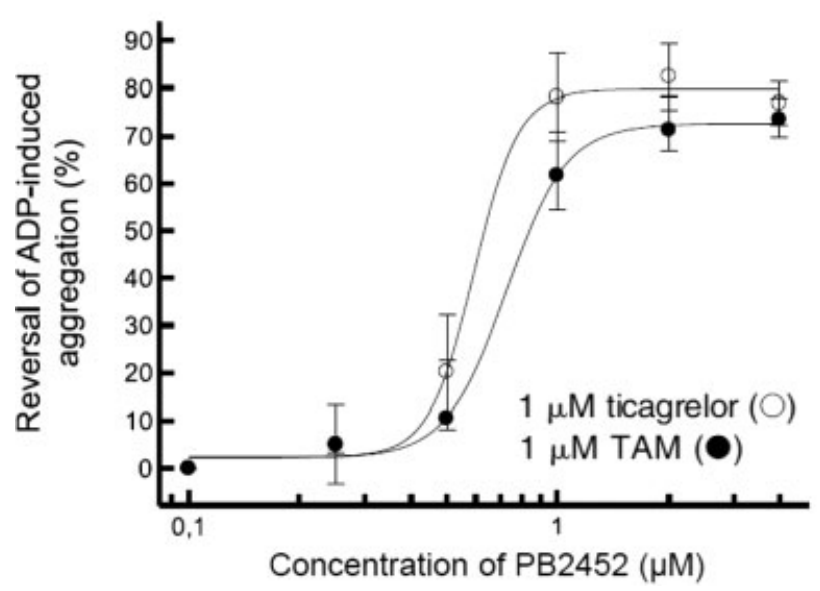

\section{Unbound ticagrelor concentration in plasma after addition of $1 \mu \mathrm{M}$ ticagrelor}

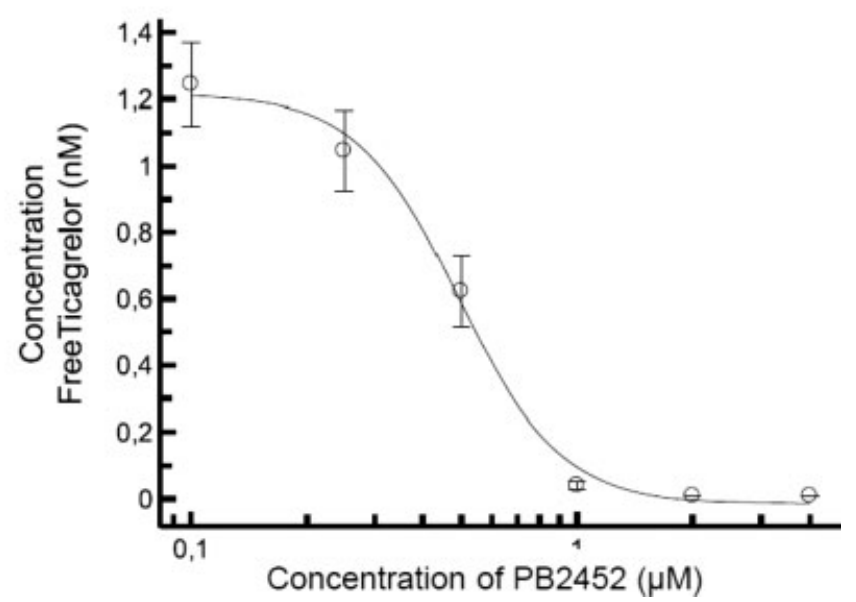

Fig. 5 In vitro concentration-response curves of the Fab fragment PB2452 on platelet inhibition by ticagrelor and the active metabolite of ticagrelor (left panel) and the reduction of unbound ticagrelor concentration (right panel). Figures adapted from Buchanan et al. ${ }^{35}$

Ten sequential dose cohorts of PB2452 were evaluated. The antibody was administered either as a 30-minute infusion (doses $0.1-9 \mathrm{~g}$ ) or by a bolus infusion followed by a prolonged infusion for between 8 and up to 16 hours (total dose of $18 \mathrm{~g}$ ). Platelet function was assessed using LTA after stimulation with 20 (5) $\mu$ M ADP, VerifyNow PRUTest, and VASP assays before and up to 48 hours after ticagrelor administration.
Subjects were pre-treated for 48 hours with ticagrelor (loading dose of $180 \mathrm{mg}$ followed by $90 \mathrm{mg}$ twice daily). A rapid but transient reversal of ticagrelor-induced platelet inhibition with 3 and $9 \mathrm{~g}$ of PB2452 was observed in LTA with a dose-dependent duration of reversal lasting 2 hours with the $9 \mathrm{~g}$ infusion. Following the bolus plus prolonged infusion regimen of $18 \mathrm{~g}$ PB2452, a significant reversal was observed 

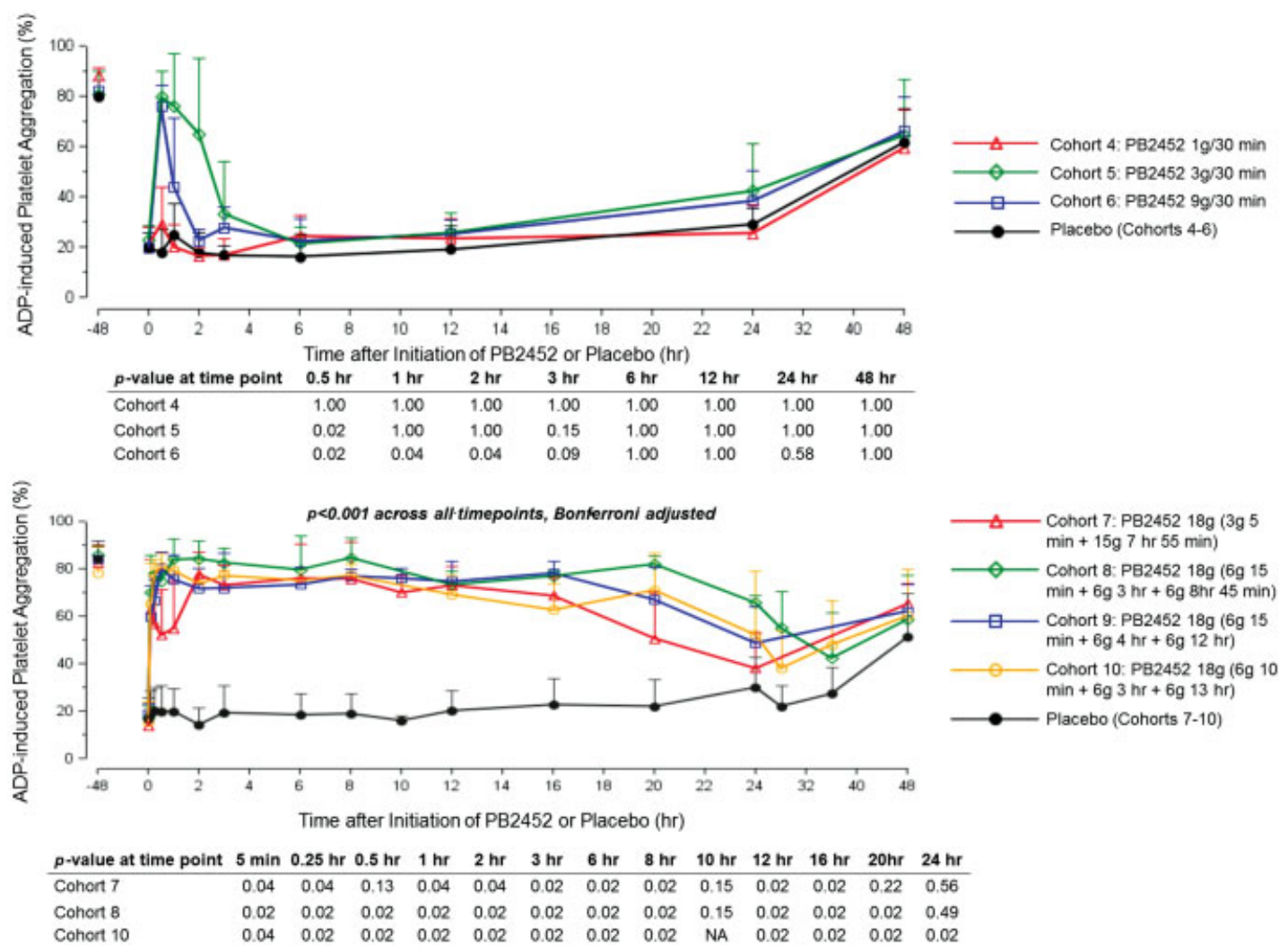

Fig. 6 Time course of the onset and offset of ticagrelor reversal by adenosine diphosphate (ADP)-induced platelet aggregation after administration of the monoclonal antibody fragment PB2452 to healthy subjects after pretreatment with ticagrelor. (A) Effect of ascending doses of PB2452 or placebo administered as a 30-minute short-term infusion. (B) Effect of an intravenous infusion of $18 \mathrm{~g}$ PB2452 or placebo administered with an infusion duration of 8,12 , or 16 hours. Figures adapted from Bhatt et al. ${ }^{38}$

5 minutes after initiation of PB2452 infusion (-Fig. 6). The duration of reversal was infusion time-dependent, lasting 20 to 24 hours with a 16-hour infusion. There was no evidence of rebound in platelet activity after cessation of PB2452. Data were consistent throughout all platelet function assays used. Adverse events related to the Fab fragment were limited mainly to issues involving the infusion site. Thus, administration of the antibody PB2452 is at present the most rapid and effective reversal strategy for the antiplatelet effect of ticagrelor.

The clinical efficacy for treatment or prevention of bleeding remains to be investigated in further studies. The reversal agent reduces blood loss in animal models of bleeding. ${ }^{37}$ Clinical data as the effective reversal of antiplatelet effect results in a more rapid restoration of hemostasis in patients bleeding on ticagrelor or the prevention of bleeding are lacking at present. If the reversal agent confirms these expectations in clinical efficacy in future studies, it would be an important advance.

\section{Conclusion}

The beneficial effect of DAPT concerning reduction of risk of ischemic events such as myocardial infarction or stent thrombosis has been shown in numerous clinical studies. In the year 2017, the concept of DAPT-which established its superiority over anticoagulant therapy among patients un- dergoing $\mathrm{PCI}-$ has its 21st anniversary since the publication of the first randomized clinical trial (ISAR Trial ${ }^{5}$ ). Based on over 35 randomized clinical trials, including more than 225,000 patients, DAPT is among the most intensively investigated treatment options in the field of cardiovascular medicine. ${ }^{17}$ The vast majority of studies focused primarily on the reduction of the risk of ischemic events including (re)infarction and stent thrombosis. On the contrary, this wellrecognized improvement in ischemic benefit by DAPT is accompanied by an increased on-treatment bleeding risk including major and fatal bleeding.

Current concepts of personalized antiplatelet therapy aim to establish alternative DAPT strategies and to preserve the benefit in prevention of ischemic events while reducing ontreatment bleeding risk. TROPICAL-ACS was the first prospective randomized study with an appropriate sample size in this context demonstrating the feasibility of an early deescalation strategy of $\mathrm{P}_{2} \mathrm{Y}_{12}$ inhibition in ACS patients guided by platelet function testing. ${ }^{39-41}$

Supplementation of platelets is the most extensively studied strategy for timely reversal of $\mathrm{P}_{2} \mathrm{Y}_{12}$ inhibition in emergency situation such as acute major bleeding or urgent surgery with high bleeding risk or unacceptable clinical consequences of bleeding. These studies investigate recovery of ex vivo determined platelet function as a surrogate for bleeding risk. Using this approach, reversal of the thienopyridine derivatives clopidogrel and prasugrel which inhibit the 
$\mathrm{P} \mathrm{Y}_{12}$ receptor for the life-span of the platelet is feasible. Data on reduction of platelet inhibition after administration of ticagrelor which has reversible binding characteristics are conflicting. Addition of human serum albumin in in vitro experiments has been shown to be an interesting alternative. Hemadsorption as an alternate approach is currently at the bench level and doubts concerning the suitability of this method in emergency situations requiring rapid reversal persist. However, besides the APTITUDE-CABG study, ${ }^{32}$ a study in healthy subjects, ${ }^{23}$ and case reports, ${ }^{33}$ the vast majority of the strategies to assess reversal strategies for $\mathrm{P}_{2} \mathrm{Y}_{12}$ inhibition are ex vivo measurements investigating increase in platelet reactivity as surrogate. Proof-of-concept studies on the clinical efficacy of these strategies on restoration of hemostasis are lacking. First dose-finding data on the use of the Fab fragment PB2452 in healthy subjects were recently published. ${ }^{38}$ It is at present the most promising option for reversal of ticagrelor, since platelet function is reversed rapidly and overall tolerability of the antibody seems to be without major concerns. The study provides promising findings but achieving longer periods of reversal required higher doses and longer infusion periods of PB2452. ${ }^{38}$ At present, we do not have any estimate on the costs for this treatment. Since U.S. Food and Drug Administration grants breakthrough therapy designation for PB2452, clinical availability might be expected in the near future.

The available in vitro studies used appropriate laboratory assays to assess the pharmacological activity on platelet function of the $\mathrm{P}_{2} \mathrm{Y}_{12}$ receptor antagonists and its reversal. Major concerns persist, if these methods reflect the interaction of platelets, the signaling cascades in the coagulation pathways, and the entire complex coagulation system to achieve hemostasis and to reduce blood loss in the clinical setting. Adequately powered clinical trials with bleeding outcome assessment would be required before definite clinical recommendations can be derived from these so far more or less experimental studies. It is more than doubtful if such trials will ever be conducted in an adequate setting, with enrollment of a sufficient number of patients and last but not least in a timely manner.

\section{Conflict of Interest}

D.T. reports lecture fees from Amgen, AstraZeneca, Bayer, Berlin Chemie, Boehringer Ingelheim, Bristol-Myers Squibb, Daiichi Sankyo, Pfizer, and Sanofi and fees for advisory board activities from Bayer, Boehringer Ingelheim, and Daiichi Sankyo. D.A. reports lecture fees from Astra Zeneca, Bayer, Boehringer Ingelheim, Pfizer, and MSD Pharma. K.S. reports personal fees from Bayer. D.S. reports grants and personal fees from Sanofi Aventis, Roche Diagnostics, and Daiichi Sankyo; personal fees from Bayer, Pfizer, and Haemonetics S.A. All the other authors have no conflict of interest to declare.

\section{References}

1 Lange RA, Hillis LD. Antiplatelet therapy for ischemic heart disease. N Engl J Med 2004;350(03):277-280

2 Lewis HD Jr, Davis JW, Archibald DG, et al. Protective effects of aspirin against acute myocardial infarction and death in men with unstable angina. Results of a Veterans Administration Cooperative Study. N Engl J Med 1983;309(07):396-403

3 Cairns JA, Gent M, Singer J, et al. Aspirin, sulfinpyrazone, or both in unstable angina. Results of a Canadian multicenter trial. N Engl J Med 1985;313(22):1369-1375

4 Théroux P, Ouimet H, McCans J, et al. Aspirin, heparin, or both to treat acute unstable angina. N Engl J Med 1988;319(17):1105-1111

5 Schömig A, Neumann FJ, Kastrati A, et al. A randomized comparison of antiplatelet and anticoagulant therapy after the placement of coronary-artery stents. N Engl J Med 1996;334(17): 1084-1089

6 Bertrand ME, Rupprecht H-J, Urban P, Gershlick AH; CLASSICS Investigators. Double-blind study of the safety of clopidogrel with and without a loading dose in combination with aspirin compared with ticlopidine in combination with aspirin after coronary stenting: the clopidogrel aspirin stent international cooperative study (CLASSICS). Circulation 2000;102(06):624-629

7 Campo G, Valgimigli M, Gemmati D, et al. Poor responsiveness to clopidogrel: drug-specific or class-effect mechanism? Evidence from a clopidogrel-to-ticlopidine crossover study. J Am Coll Cardiol 2007;50(12):1132-1137

8 Gurbel PA, Bliden KP, Hiatt BL, O'Connor CM. Clopidogrel for coronary stenting: response variability, drug resistance, and the effect of pretreatment platelet reactivity. Circulation 2003;107 (23):2908-2913

9 Hochholzer W, Trenk D, Bestehorn HP, et al. Impact of the degree of peri-interventional platelet inhibition after loading with clopidogrel on early clinical outcome of elective coronary stent placement. J Am Coll Cardiol 2006;48(09):1742-1750

10 Aradi D, Komócsi A, Vorobcsuk A, et al. Prognostic significance of high on-clopidogrel platelet reactivity after percutaneous coronary intervention: systematic review and meta-analysis. Am Heart J 2010;160(03):543-551

11 Sibbing D, Braun S, Morath T, et al. Platelet reactivity after clopidogrel treatment assessed with point-of-care analysis and early drugeluting stent thrombosis. J Am Coll Cardiol 2009;53(10):849-856

12 Parodi G, Marcucci R, Valenti R, et al. High residual platelet reactivity after clopidogrel loading and long-term cardiovascular events among patients with acute coronary syndromes undergoing PCI. JAMA 2011;306(11):1215-1223

13 Wiviott SD, Braunwald E, McCabe $\mathrm{CH}$, et al; TRITON-TIMI 38 Investigators. Prasugrel versus clopidogrel in patients with acute coronary syndromes. N Engl J Med 2007;357(20):2001-2015

14 Wallentin L, Becker RC, Budaj A, et al; PLATO Investigators. Ticagrelor versus clopidogrel in patients with acute coronary syndromes. N Engl J Med 2009;361(11):1045-1057

15 Levine GN, Bates ER, Bittl JA, et al. 2016 ACC/AHA guideline focused update on duration of dual antiplatelet therapy in patients with coronary artery disease: a report of the American College of Cardiology/American Heart Association Task Force on Clinical Practice Guidelines. J Am Coll Cardiol 2016;68(10): $1082-1115$

16 Neumann F-J, Sousa-Uva M, Ahlsson A, et al; ESC Scientific Document Group. 2018 ESC/EACTS Guidelines on myocardial revascularization. Eur Heart J 2019;40(02):87-165

17 Valgimigli M, Bueno H, Byrne RA, et al; ESC Scientific Document Group; ESC Committee for Practice Guidelines (CPG); ESC National Cardiac Societies. 2017 ESC focused update on dual antiplatelet therapy in coronary artery disease developed in collaboration with EACTS: the Task Force for dual antiplatelet therapy in coronary artery disease of the European Society of Cardiology (ESC) and of the European Association for Cardio-Thoracic Surgery (EACTS). Eur Heart J 2018;39(03):213-260

18 Egholm G, Kristensen SD, Thim T, et al. Risk associated with surgery within 12 months after coronary drug-eluting stent implantation. J Am Coll Cardiol 2016;68(24):2622-2632

19 Akers WS, Oh JJ, Oestreich JH, Ferraris S, Wethington M, Steinhubl SR. Pharmacokinetics and pharmacodynamics of a bolus and 
infusion of cangrelor: a direct, parenteral P2Y12 receptor antagonist. J Clin Pharmacol 2010;50(01):27-35

20 Ferri N, Corsini A, Bellosta S. Pharmacology of the new P2Y12 receptor inhibitors: insights on pharmacokinetic and pharmacodynamic properties. Drugs 2013;73(15):1681-1709

21 Sillén H, Cook M, Davis P. Determination of unbound ticagrelor and its active metabolite (AR-C124910XX) in human plasma by equilibrium dialysis and LC-MS/MS.J Chromatogr B Analyt Technol Biomed Life Sci 2011;879(23):2315-2322

22 Vilahur G, Choi BG, Zafar MU, et al. Normalization of platelet reactivity in clopidogrel-treated subjects. J Thromb Haemost 2007;5(01):82-90

23 Prüller F, Drexler C, Archan S, Macher S, Raggam RB, Mahla E. Low platelet reactivity is recovered by transfusion of stored platelets: a healthy volunteer in vivo study. J Thromb Haemost 2011;9(08): 1670-1673

24 Zafar MU, Santos-Gallego C, Vorchheimer DA, et al. Platelet function normalization after a prasugrel loading-dose: timedependent effect of platelet supplementation. J Thromb Haemost 2013;11(01):100-106

25 Bonhomme F, Bonvini R, Reny JL, Poncet A, Fontana P. Impact of non-inhibited platelet supplementation on platelet reactivity in patients treated with prasugrel or ticagrelor for an acute coronary syndrome: an ex vivo study. Platelets 2015;26(04):324-330

26 Hobl EL, Derhaschnig U, Firbas C, Schoergenhofer C, Schwameis M, Jilma B. Reversal strategy in antagonizing the P2Y12 -inhibitor ticagrelor. Eur J Clin Invest 2013;43(12):1258-1261

27 Hansson EC, Shams Hakimi C, Åström-Olsson K, et al. Effects of ex vivo platelet supplementation on platelet aggregability in blood samples from patients treated with acetylsalicylic acid, clopidogrel, or ticagrelor. Br J Anaesth 2014;112(03):570-575

28 Zafar MU, Smith DA, Baber U, et al. Impact of timing on the functional recovery achieved with platelet supplementation after treatment with ticagrelor. Circ Cardiovasc Interv 2017;10(08):e005120

29 Kruger P, Chan N, Eikelboom JW. Platelet transfusion for ticagrelor reversal. Circ Cardiovasc Interv 2017;10(08):e005579

30 Scharbert G, Wetzel L, Schrottmaier WC, Kral JB, Weber T, Assinger A. Comparison of patient intake of ticagrelor, prasugrel, or clopidogrel on restoring platelet function by donor platelets. Transfusion 2015;55(06):1320-1326

31 Schoener L, Jellinghaus S, Richter B, et al. Reversal of the platelet inhibitory effect of the $\mathrm{P}_{2} \mathrm{Y}_{12}$ inhibitors clopidogrel, prasugrel, and ticagrelor in vitro: a new approach to an old issue. Clin Res Cardiol 2017;106(11):868-874

32 O'Connor SA, Amour J, Mercadier A, et al; ACTION Study Group. Efficacy of ex vivo autologous and in vivo platelet transfusion in the reversal of P2Y12 inhibition by clopidogrel, prasugrel, and ticagrelor: the APTITUDE study. Circ Cardiovasc Interv 2015;8(11):e002786

33 Godier A, Taylor G, Gaussem P. Inefficacy of platelet transfusion to reverse ticagrelor. N Engl J Med 2015;372(02):196-197

34 Angheloiu GO, Gugiu GB, Ruse C, Pandey R, Dasari RR, Whatling C. Ticagrelor removal from human blood. JACC Basic Transl Sci 2017; 2(02):135-145

35 Buchanan A, Newton P, Pehrsson S, et al. Structural and functional characterization of a specific antidote for ticagrelor. Blood 2015; 125(22):3484-3490

36 Erlinge D. The first specific antiplatelet antidote. Blood 2015;125 (22):3372-3374

37 Pehrsson S, Johansson KJ, Janefeldt A, et al. Hemostatic effects of the ticagrelor antidote MEDI2452 in pigs treated with ticagrelor on a background of aspirin. J Thromb Haemost 2017;15(06):1213-1222

38 Bhatt DL, Pollack CV, Weitz JI, et al. Antibody-based ticagrelor reversal agent in healthy volunteers. N Engl J Med 2019;380(19): $1825-1833$

39 Sibbing D, Aradi D, Jacobshagen C, et al; TROPICAL-ACS Investigators. Guided de-escalation of antiplatelet treatment in patients with acute coronary syndrome undergoing percutaneous coronary intervention (TROPICAL-ACS): a randomised, open-label, multicentre trial. Lancet 2017;390(10104):1747-1757

40 Aradi D, Gross L, Trenk D, et al. Platelet reactivity and clinical outcomes in acute coronary syndrome patients treated with prasugrel and clopidogrel: a pre-specified exploratory analysis from the TROPICAL-ACS trial. Eur Heart J 2019;40(24):1942-1951

41 Sibbing D, Aradi D, Jacobshagen C, et al; TROPICAL-ACS Investigators. A randomised trial on platelet function-guided de-escalation of antiplatelet treatment in ACS patients undergoing $\mathrm{PCI}$. Rationale and design of the Testing Responsiveness to Platelet Inhibition on Chronic Antiplatelet Treatment for Acute Coronary Syndromes (TROPICAL-ACS) Trial. Thromb Haemost 2017;117 (01):188-195

42 Small DS, Li YG, Ernest CS II, et al. Integrated analysis of pharmacokinetic data across multiple clinical pharmacology studies of prasugrel, a new thienopyridine antiplatelet agent. J Clin Pharmacol 2011;51(03):321-332 